\title{
Subsidence of anchored cage after anterior cervical discectomy
}

\author{
Opsenak R, Hanko M, Snopko P, Varga K, Kolarovszki B \\ Clinic of Neurosurgery, Jessenius Faculty of Medicine in Martin, Comenius University in Bratislava, \\ University Hospital Martin, Slovakia. branislav.kolarovszki@gmail.com
}

\begin{abstract}
PURPOSE: Cage subsidence (CS) represents a risk factor for adjacent segment degeneration (ASD) and unfavorable results of anterior cervical discectomy (ACD).

METHODS: Sixty-one patients after level 1 or 2 of ACD with implantation of Zero Profile VA cage were included in the study. CS was assessed with a follow-up period of 12 months after ACD. The impacts of factors such as sex, age, number of operated segments, osteoporosis and extent of peroperative distraction were assessed in relation to the incidence of CS. The influences of CS on clinical results (VAS, NDI, Odom's criteria) and ASD incidence were evaluated.

RESULTS: In $74 \%$ of cases there was the presence of CS into both adjacent vertebral bodies. CS into the ventral part of motion segment was dominant during the entire follow-up period. CS had no influence on clinical results of $A C D$. Parallel CS into both ventral and dorsal parts of motion segments significantly increased the incidence of proximal ASD $(p=0.0163)$. Osteoporosis and extent of peroperative distraction were linked to higher incidence of CS into the dorsal part of motion segment $(p<0.05)$.

CONCLUSIONS: Osteoporosis and the extent of peroperative distraction are risk factors for the subsidence of anchored cage and while increasing the incidence of proximal adjacent segment degeneration it has no significant influence on clinical results of surgery (Tab. 3, Fig. 5, Ref. 32). Text in PDF www.elis.sk.

KEY WORDS: cage subsidence, cervical discectomy, risk factors, complications, adjacent segment degeneration.
\end{abstract}

\section{Introduction}

Cage subsidence (CS) represents a breakthrough of intervertebral disc replacement into the trabecular bone of adjacent vertebral bodies with a consecutive decrease in the height of intervertebral space. Risk factors for CS include osteoporosis, extent of peroperative intervertebral space distraction, improper position of the implant in the intervertebral space, size of the contact plane between the implant and adjacent vertebral body endplate, extent of intraoperative vertebral endplate treatment, excessive resection of ventral osteophytes, implant fixated by means of a ventral plate, and type of material used for intervertebral cage production (1-9). CS after an anterior cervical discectomy (ACD) occurs most often in the ventral part of the motion segment (VPMS) thus resulting in segmental kyphosis which is a risk factor for the adjacent segment degeneration (ASD) $(1,10)$. CS can even lead to restenosis of neuroforamina and therefore to the relapse of the previous ra-

Clinic of Neurosurgery, Jessenius Faculty of Medicine in Martin, Comenius University in Bratislava, University Hospital Martin, Slovakia

Address for correspondence: $\mathrm{B}$. Kolarovszki, MD, PhD, Clinic of Neurosurgery, Jessenius Faculty of Medicine in Martin, Comenius University in Bratislava, University Hospital Martin, Kollarova 2, SK-036 59 Martin, Slovakia.

Phone: +421.43 .4203903$

Acknowledgements: This work was supported by project named The application of PACS (Picture Archiving and Communication System) in research and development, ITMS 26210120004. dicular symptomatology. An intervertebral cage anchored by means of fixating screws carries a presumption of higher incidence of its subsidence when compared to intervertebral cages secured by a conventional plate $(7,11-14)$.

\section{Material and methods}

This prospective study includes 61 patients with a degenerative disc disease of the cervical spine who underwent a microsurgical 1- or 2-level ACD during the period from May 2013 to April 2015. The patients with correlating clinical and graphic findings were indicated for surgical treatment after exhaustion of conservative treatment for a minimum duration of 6 weeks. In all cases included, the surgically treated motion segments showed graphic signs of osteochondrosis. As a replacement of the intervertebral disc, a single type of anchored intervertebral cage was used, namely Zero Profile Variable Angle (DePuy Synthes, Switzerland, Zero$P$ VA). This type of cage is fixed in the intervertebral space by means of two divergently introduced screws (Fig. 1). During the cervical discectomy we aimed for the maximum preservation of the integrity of adjacent vertebral endplates. We used exclusive criteria as follows: myelopathy, pregnancy, presence of inflammatory or oncological disease of the cervical spine, cervical spine trauma, and any type of contraindication for the elective surgery. As for the age of patients included, the cohort was divided into two subgroups, namely patients younger than 55years and those aged 55 years or older. The cohort was also divided according to patients' sex and number of operated segments while there was 

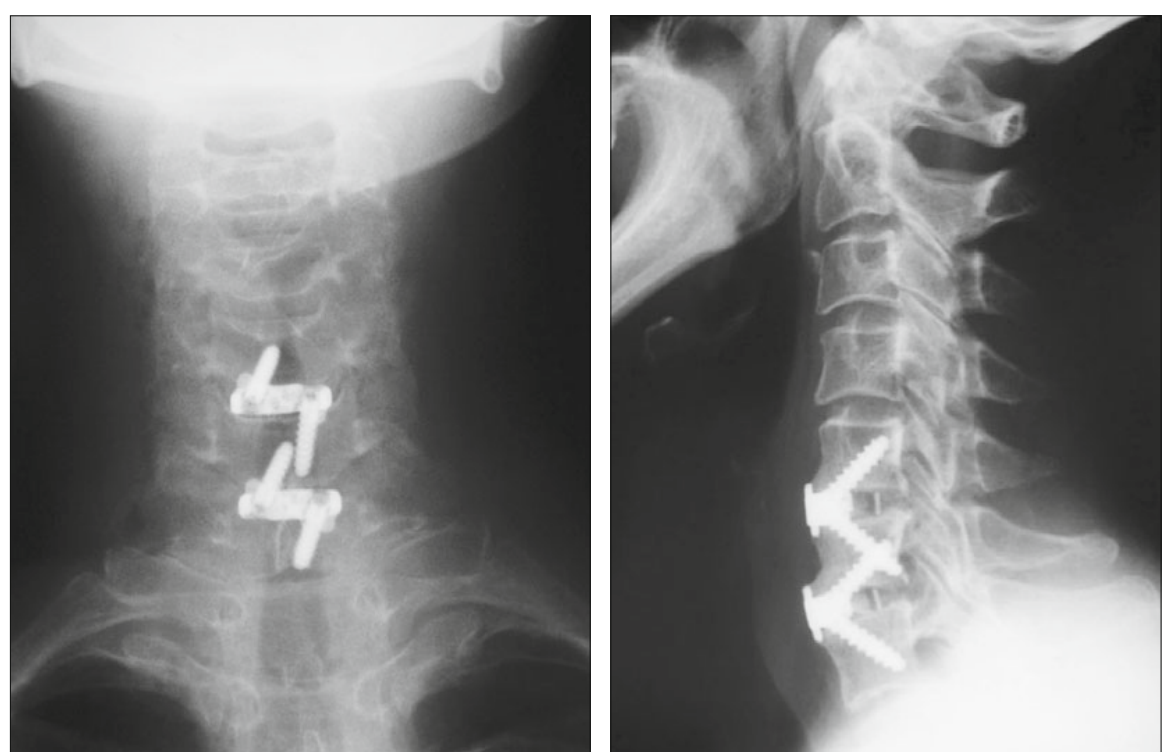

Fig. 1. X-ray of cervical spine after Zero-P cage implantation (authors's archive).

to us, a Slovak mutation of the questionnaire was created by translating the English version (15). The outcome of surgical treatment was also evaluated objectively by means of Odom's criteria as part of postoperative clinical controls performed by the surgeon (16).

In all patients, radiographic examinations in anteroposterior and lateral projections were carried out preoperatively, early into the postoperative period (within first 48 hours) and as part of follow-ups performed 6 weeks, 3 months, 6 months and 12 months after the surgery. The height of intervertebral space was measured indirectly as a relative height of ventral and dorsal parts of the motion segment. The relative height of VPMS was measured as a ratio of the height of motion segment in the sagittal plane measured at the

also a subgroup of patients with a densitometrically verified diagnosis of osteoporosis.

Amongst the prospectively evaluated clinical factors there was a subjective assessment of life quality using the neck disability index (NDI) questionnaire and assessment of pain intensity separately for neck (VAS C) and upper limbs (VAS UL) while using the visual analogue scale preoperatively and postoperatively with a follow-up of 6 weeks, 3 months, 6 months and 12 months. Since the official Slovak form of the NDI questionnaire was not available

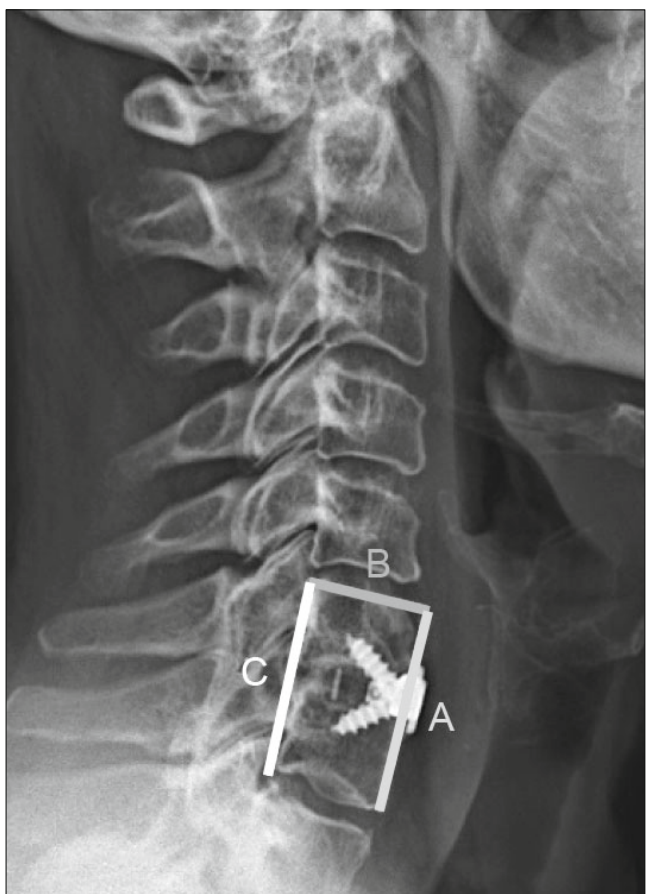

Fig. 2. Method of measuring the relative height of the ventral (A/B) and dorsal (C/D) motion segment parts level of anterior margins of vertebral bodies to the length of upper endplate of the proximal vertebral body. Similarly, the dorsal part of the motion segment (DPMS) was assessed as a ratio of the motion segment's height measured at the level of posterior margins of the vertebral bodies to the length of upper endplate of the proximal vertebral body (Fig. 2). The relative heights of motion segments were also measured preoperatively, early postoperatively (within first 48 hours) and as part of follow-ups performed 6 weeks, 3 months, 6 months and 12 months after the surgery.

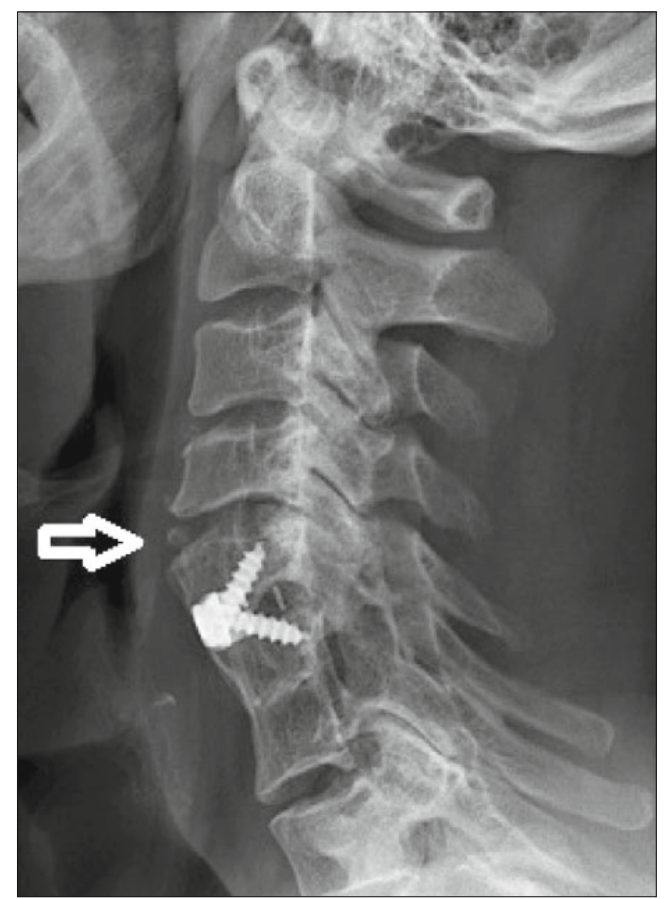

Fig. 3. Adjacent segment degeneration $\mathrm{C} 4 / 5$ in form of incrustation of anterior longitudinal ligament (authors's archive). 


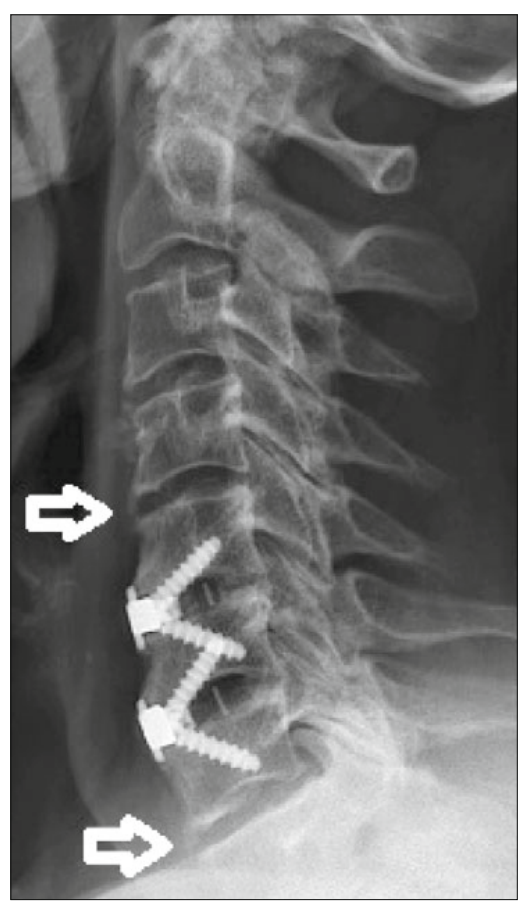

Fig. 4. Proximal and distal adjacent segment degeneration in form of the formation of ventral osteophytes (authors's archive).

CS was defined as a decrease in the relative motion segment's height, namely by $10 \%$ and more when compared to the early postoperative finding. The impact of factors such as patients' sex, age, number of operated segments and osteoporosis on the incidence of CS was statistically evaluated using the Fischer's exact test. Twelve months after ACD, the effect of CS was evaluated with regards to the efficiency of the surgical treatment (VAS C, VAS UL, NDI, Odom's criteria). This was done both separately for VPMS, and DPMS, and concurrently for both sides. The significance of this effect was evaluated by means of the Student's unpaired t-test. The relationship of the extent of distraction of VPMS and DPMS in the early postoperative period to the incidence of CS was evaluated using the Student's unpaired t-test. A value of $\mathrm{p}<0.05$ was defined as statistically significant.

The degeneration of the motion segment, adjacent to the segment treated by ACD, was analyzed using the radiographic findings obtained 12 months after the surgery. Radiographic findings of ASD (Figs 2, 3 and 4) included incrustation of the anterior longitudinal ligament, development or progression of ventral or dorsal osteophytes or degenerative listhesis. The degeneration of adjacent segment and its eventual clinical manifestation was evaluated separately for proximal and distal motion segments. The

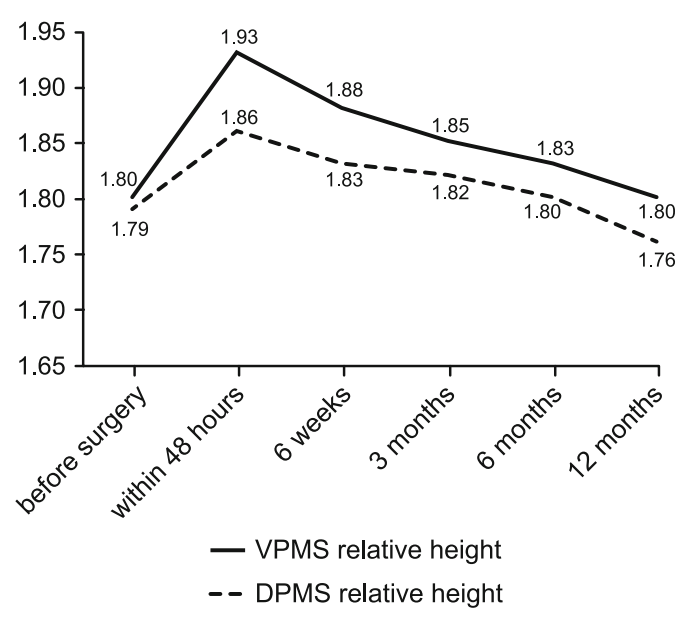

Fig. 5. Time-evolving graph of motion segment's relative height before and after the surgery.

impact of factors such as patients' sex, age, number of operated segments, osteoporosis and CS on the incidence of ASD was statistically evaluated using the Fischer's exact test with a value of $\mathrm{p}<0.05$, thus defined as being statistically significant.

\section{Results}

In our study, data of 61 patients were assessed and 94 intervertebral levels of the cervical spine were treated in total. The most commonly treated levels of the cervical spine were C5/6 and C6/7.

There was a significant increase in the relative height of VPMS and DPMS in the early period after cage implantation. The increase in VPMS was significantly higher than the increase in DPMS in the early postoperative period $(\mathrm{p}=0.0002)$. There was a significant decrease in VPMS compared to DPMS at 3 months, 6 months and 12 months into the follow-up period after ACD $(\mathrm{p}<0.05)$. The average relative height of VPMS at 12 months into the follow-up period after ACD was very similar to preoperative findings. The average height of DPMS was lower compared to preoperative findings (Fig. 5).

The incidence of CS in our group of patients is shown in Table 1. The incidence of isolated CS in VPMS was present in $23 \%$ of patients at 12 months into the follow-up period after ACD. CS in VPMS and DPMS was present in $21 \%$ of patients at 12 months into the follow-up period after ACD. The incidence of isolated CS in DPMS was present in $11.5 \%$ of patients at 12 months into the follow-up period after ACD. Total incidence of CS was $18 \%$ at 6 weeks into the follow-up period and at 12 months, it increased up to $56 \%$. CS into both adjacent vertebral bodies was present

Tab. 1. Incidence of cage subsidence in the study (calculated per patient/treated segment).

\begin{tabular}{|c|c|c|c|c|}
\hline Cage subsidence & VPMS & DPMS & VPMS + DPMS & Total incidence \\
\hline 6 weeks & $11.4 \% / 7.4 \%$ & $0 \% / 0 \%$ & $6.6 \% / 4.3 \%$ & $18 \% / 11.7 \%$ \\
\hline 3 months & $16.4 \% / 12.8 \%$ & $3.3 \% / 2.1 \%$ & $13.1 \% / 8.5 \%$ & $32.8 \% / 23.4 \%$ \\
\hline 6 months & $14.7 \% / 11.7 \%$ & $8.2 \% / 5.4 \%$ & $16.4 \% / 10.6 \%$ & $39.3 \% / 27.7 \%$ \\
\hline 12 months & $23 \% / 19.2 \%$ & $11.5 \% / 7.5 \%$ & $21.3 \% / 17 \%$ & $55.8 \% / 43.7 \%$ \\
\hline
\end{tabular}


Tab. 2. Development of neck pain, pain of upper extremities, and quality of life after ACD.

\begin{tabular}{|c|c|c|c|c|c|}
\hline Clinical results & Before & 6 weeks & 3 months & 6 months & 12 months \\
\hline VAS C & 5.39 & $3.85 /-28.6 \%$ & $3.84 /-28.8 \%$ & $3.69 /-31.5 \%$ & $3.77 /-30.1 \%$ \\
\hline VAS UL & 5.82 & $3.08 /-47.1 \%$ & $3.54 /-39.2 \%$ & $3.36 /-42.3 \%$ & $3.41 /-41.4 \%$ \\
\hline NDI & 48.4 & $40.1 /-17.1 \%$ & $39.3 /-18.8 \%$ & $38.5 /-20.5 \%$ & $37.8 /-21.9 \%$ \\
\hline
\end{tabular}

Tab. 3. Graphical manifestation of adjacent segment degeneration in the study.

\begin{tabular}{lc}
\hline Morphological types of ASD & $\begin{array}{c}\text { Representation in } \\
\text { the study }\end{array}$ \\
\hline calcification of the anterior longitudinal ligament $4 / 23.5 \%$ \\
formation of ventral osteophyte & $9 / 52.9 \%$ \\
degenerative spondylolisthesis & $2 / 11.8 \%$ \\
formation of dorsal osteophyte & $1 / 5.9 \%$ \\
degenerative instability & $1 / 5.9 \%$ \\
\hline
\end{tabular}

in $74 \%$ of treated levels, while the subsidence to the distal cervical vertebral body was present in $26 \%$ of treated levels. In our group of patients there was no isolated CS into the proximal vertebral body.

The preoperative and postoperative results of VAS C, VAS UL and NDI in our group of patients are compared in Table 2. In the postoperative period there was a decrease in neck pain and radicular pain in the upper limbs while the patients' life quality improved. Throughout the follow-up period, clinical outcomes according to Odom's criteria were evaluated as being "good" (minimal persistence of preoperative symptoms, abnormal findings improved). CS did not have any significant impact on clinical outcomes after the surgery.

The incidence of ASD was $27.9 \%$, with a dominant disability of proximal adjacent segment (14.8\%). Clinical symptoms of ASD were present in 3 patients (17.6\%). All patients with symptomatic ASD were indicated to surgical procedure, namely discectomy and fusion. In all cases of ASD surgery, degeneration of distal segment after previous ACD was present. Graphical manifestation of ASD is shown in Table 3. The most common graphical manifestation was that of the formation of ventral osteophyte (no difference between distal and proximal adjacent segments). The calcification of anterior longitudinal ligament had the form of incrustation. In cases of isolated CS in DPMS, the incidence of ASD was higher, but without statistical significance. CS in VPMS and DPMS had a statistically significant impact on the incidence of proximal $\operatorname{ASD}(p=0.0163)$, but the cases were without clinical symptoms.

In the group of patients with osteoporosis, the incidence of CS was higher in both VPMS and DPMS, but the significance ( $p$ $<0.05$ ) was found only in the incidence of the latter. There was no correlation between age, sex, number of operated segments and incidence of CS.

When compared to patients without CS, the group of patients with CS in VPMS (both VPMS and DPMS), yielded a higher early postoperative distraction in VPMS, but the results were without statistical significance. When compared to patients without CS, the group of patients with CS in DPMS yielded also a higher early postoperative distraction in DPMS, while the results were statistically significant $(\mathrm{p}<0.01)$.
When compared to patients without CS, the groups of patients with CS in both VPMS and DPMS yielded higher early postoperative distraction in VPMS and DPMS, respectively, but the significance $(p<0.001)$ was found only in the incidence of the latter.

\section{Discussion}

The incidence of CS after ACD is reported in range of 0-40\% $(1,7,11,14,17-22)$. Bartels et al report the incidence of CS without plate fixation at $29.2 \%$ (17). Wu et al even report the incidence of CS up to $19.1 \%$ (19). Dhir et al report the subsidence of Zero Profile (DePuy Synthes, Switzerland) anchored cage in all cases of implantation. At the same time however, they consider CS significant when it reaches $3 \mathrm{~mm}$ and more, and in those cases, CS had an incidence of $2 \%$ (11). In our study, the subsidence incidence of Zero-P VA cage was at $56 \%$ at 12 months after the surgery.

Many studies report the incidence of subsidence of anchored cage to be higher compared to cages fixed with a conventional plate $(7,12-14,23-25)$. Other published studies did not confirm this premise (26-28). Chen et al report the incidence of titanium cage subsidence to be higher compared to that of polyetheretherketone (PEEK) cages (2). In our study, the incidence of CS at 12 months after ACD was high despite the fact that most of the Zero-P VA cages are made of PEEK and the cage was fixed by two screws (29). In our study, CS did not have a significant impact on the effect of surgical treatment. Similar findings are also reported in other published studies $(1,7,13,14,19-21,30)$.

In our study, osteoporosis was a risk factor of CS. It had a significant impact on the subsidence in DPMS. It was therefore confirmed that the weakening of the mechanical resistance of the bone significantly affected the incidence of CS $(4,8)$. Similarly, an extensive peroperative treatment of endplates of the adjacent vertebral bodies is one of the risk factors of CS $(1,9)$. In their biomechanical studies on cadavers, Nagaraja et al found out that the treatment of endplates leads to their thinning by an average of $20 \%$. An extensive treatment of endplates has led to the weakening of cages with an integrated plate (5). In our study, during the surgery, we tried to preserve the maximum integrity of the endplates of adjacent vertebral body. Nevertheless, our incidence of CS was at the level of $56 \%$.

In our data, CS into both adjacent vertebral bodies was present in up to $74 \%$ of cases. In the remaining cases, it was present only in the distal adjacent body of the vertebra. Isolated CS into the proximal adjacent vertebra did not occur in our study. Barsa and Suchomel report yet another tendency. In their study, almost $90 \%$ of cases had CS in the distal adjacent vertebra. The authors implanted a stand-alone cage and all cases of CS were present in VPMS (1). CS in VPMS (23\%) was predominant also in our study. 
356-361

The biomechanical stability of the Zero Profile cage, the design of which is similar to that of the cage used in our set, is limited in anteflexion and retroflexion (31). Unlike the Zero Profile cage, the Zero-P VA cage is fixed with only two screws. Postoperative segmental micro-movements are considered to be one of the causes of CS $(1,32)$. The range of peroperative distraction in our study was a risk factor for CS. Distraction in DPMS was statistically significant. Truumees et al demonstrated that the size of bone graft is in significant direct proportion with the strength of distraction forces that increase the graft compression during insertion (6). The range of peroperative distraction is considered to be a significant risk factor for CS and ASD (3). Other published studies did not confirm this logical premise $(1,19)$. CS in VPMS leads to segmental kyphosis, which is a risk factor of ASD (10). In our study, isolated CS in VPMS did not have a significant impact on the incidence of ASD. However, CS in both, VPMS and DPMS had a significant impact on the incidence of ASD.

\section{Conclusion}

The incidence of anchored CS was high despite its fixation by two screws. CS occurred most often in VPMS without being significantly affected by observed risk factors and comorbidities. The risk factors of CS in DPMS included a densitometrically verified diagnosis of osteoporosis and extent of peroperative distraction of the surgically treated segment. CS had no significant impact on the clinical outcome after the surgery. CS simultaneously present in VPMS and DPMS was associated with a higher incidence of proximal ASD. Proximal ASD was not symptomatic in either of cases. Only distal ASD was clinically manifested.

\section{References}

1. Barsa $\mathbf{P}$, Suchomel P. Factors affecting sagittal malalignment due to cage subsidence in standalone cage assisted anterior cervical fusion.Eur Spine J 2007; 16: 1395-1400.

2. Chen Y, Wang X, Lu X, Yang L, Yang H, Yuan W, Chen D. Comparison of titanium and polyetheretherketone (PEEK) cages in the surgical treatment of multilevel cervical spondylotic myelopathy: a prospective, randomized, control study with over 7-year follow-up. Eur Spine J 2013; 22 (7): 1539-1546

3. Li J, Li Y, Kong F, Zhang D, Zhang Y, Shen Y.Adjacent segment degeneration after single-level anterior cervical decompression and fusion: disc space distraction and its impact on clinical outcomes. J Clin Neurosci 2015; 22 (3): 566-569.

4. Lim TH, Kwon H, Jeon CH, Kim JG, Sokolowski M, Natarajan R, An HS, Andersson GB. Effect of endplate conditions an bone mineral density on the compressive strengt of the graft-endplate interface in anterior cervical spine fusion. Spine (Phila Pa 1976) 2001; 26: 951-956.

5. Nagaraja S, Palepu V, Peck JH, Helgeson MD. Impact of screw location and endplate preparation on pullout strength for anterior plates and integrated fixation cages. Spine (Phila Pa 1976) 2015; 15 (11): 2425-2432.

6. Truumees E, Demetropoulos CK, Yang KH, Herkowitz HN. Effects of disc distractive forces on graft compression in an anterior cervical discectomy model. Spine (Phila Pa 1976) 2002; 27: 2441-2445.
7. Lu Y, Bao W, Wang Z, Zhou F, Zou J, Jiang W, Yang H, Zhang Z, Zhu X. Comparison of the clinical effects of zero-profile anchored spacer (ROI-C) and conventional cage-plate construct for the treatment of noncontiguous bilevel of cervical degenerative disc disease (CDDD): A minimum 2-year follow-up. Medicine (Baltimore) 2018; 97 (5): e9808.

8. Seo DK, Kim MK, Choi SJ, Sohn JY, Kim YK, Jeong EK, Ha JK, Kim CH, Park JH. Can an Anchored Cage be Substituted for an Anterior Cervical Plate and Screw for Single-Level Anterior Cervical Fusion Surgery? Prediction of Poor Candidates Through a Review of Early Clinical and Radiologic Outcomes. Clin Spine Surg 2017; 30 (9): E1289-E1297.

9. Zhang B, Li S, Miao D, Zhao C, Wang L. Risk Factors of Cage Subsidence in Patients with Ossification of Posterior Longitudinal Ligament (OPLL) After Anterior Cervical Discectomy and Fusion. Med Sci Monit 2018; 9 (24): 4753-4759.

10. Katsuura A, Hukuda S, Suruhashi Y, Mori K. Kyphotic malaligment after anterior cervical fusion is one of the factors promoting the degenerative process in adjacent intervertebral levels. Eur Spine J 2001; 10: $320-324$.

11. Dhir J, Carpenter C, Pamde R, Pyrovolou N, Ahuja S. Radiological outcome of use of Zero P cage in cervical stabilization - short term study. J Bone Joint Surg Br 2012; 94 (Suppl): 142.

12. Lee YS, Kim YB, Park SW.Does a zero-profile anchored cage offer additional stabilization as anterior cervical plate? Spine (Phila Pa 1976) 2015; 40 (10): 563-570.

13. Chen Y, Lü G, Wang B, Li L, Kuang L. A comparison of anterior cervical discectomy and fusion (ACDF) using self-locking stand-alone polyetheretherketone (PEEK) cage with ACDF using cage and plate in the treatment of three-level cervical degenerative spondylopathy: a retrospective study with 2-year follow-up. Eur Spine J 2016; 25 (7): 2255-2262.

14. Shi S, Liu ZD, Li XF, Qian L, Zhong GB, Chen FJ. Comparison of plate-cage construct and stand-alone anchored spacer in the surgical treatment of three-level cervical spondylotic myelopathy: a preliminary clinical study. Spine J 2015; 15 (9): 1973-1980.

15. Vernon H, Mior S. The Neck Disability Index: a study of reliability and validity. J Manipulative Physiol Ther 1991; 14 (7): 409-415.

16. Odom G. L., Finney W., Woodhall B. Cervical disc lesions. JAMA 1958; 166: 23-28.

17. Bartels RH, Donk RD, Feuth T. Subsidence of stand alone cervical carbon fibers cages. Neurosurgery 2005; 58: 502-508.

18. Filip M, Linzer P, Samal F, Jurek P, Strand Z, Strand J.Bioactive titan cage Implaspin in treatment of degenerative disease of the cervical spine-the results from 2007 till 2008. Chir Narzadów Ruchu i Ortopedia Polska 2010; 75 (1): 69-73.

19. Wu WJ, Jiang LS, Liang Y, Dai LY. Cage subsidence does not, but cervical lordosis improvement does affect the long-term results of anterior cervical fusion with stand-alone cage for degenerative cervical disc disease: a retrospektive study. Eur Spine J 2012; 21 (7): 1374-1382.

20. Lonjon N, Favreul E, Huppert J, Lioret E, Delhaye M, Mraidi R. Clinical and radiological outcomes of a cervical cage with integrated fixation. Medicine (Baltimore) 2019; 98 (3): e14097.

21. Ng EP, Yip AS, Wan KH, Tse MS, Wong KK, Kwok TK, Wong WC. Stand-Alone Cervical Cages in 2-Level Anterior Interbody Fusion in Cervical Spondylotic Myelopathy: Results from a Minimum 2-Year Follow-up. Asian Spine J 2018 [Epub ahead of print]. 
22. Noordhoek I, Koning MT, Jacobs WCH, Vleggeerh-Lankamp CLA. Incidence and clinical relevance of cage subsidence in anterior cervical discectomy and fusion: a systematic review. Acta Neurochir (Wien) 2018; 160 (4): 873-880.

23. Liu W, Hu L, Wang J, Liu M, Wang X. Comparison of zero-profile anchored spacer versus plate-cage construct in treatment of cervical spondylosis with regard to clinical outcomes and incidence of major complications: a meta-analysis. Ther Clin Risk Mang 2015; 23 (11): 1437-1447.

24. Zhu D, Zhang D, Liu B, Li C, Zhu J. Can Self-Locking Cages Offer the Same Clinical Outcomes as Anterior Cage-with-Plate Fixation for 3-Level Anterior Cervical Discectomy and Fusion (ACDF) in Mid-Term Follow-Up? Med Sci Monit 2019; 25: 547-557.

25. Lu VM, Mobbs RJ, Fang B, Phan K. Clinical outcomes of locking stand-alone cage versus anterior plate construct in two-level anterior cervical discectomy and fusion: a systematic review and meta-analysis. Eur Spine J 2018 [Epub ahead of print].

26. Nemoto O, Kitada A, Naitou S, Tachibana A, Ito Y, Fujikawa A. Stand- alone anchored cage versus cage with plating for single-level anterior cervical discectomy and fusion: a prospective, randomized, controlled study with a 2-year follow-up. Eur J Orthop Surg Traumatol 2015; 25 (Suppl 1): S127-S134.

27. Zhang D, Zhu D, Zhu J, Li C, Wei F, Yuan Y, Liu B. Comparison of Clinical and Radiological Outcomes Between Self-Locking StandAlone Cage and Cage with Anterior Plate for Multilevel Anterior Cervical Discectomy and Fusion: A Meta-Analysis. World Neurosurg 2019; pii: S1878-8750 (19): 30101-30109. [Epub ahead of print].
28. Shen Y, Du W, Wang LF, Dong Z, Wang F. Comparison of Zeroprofile Device versus Plate-and-Cage Implant in the Treatment of Symptomatic Adjacent Segment Disease after Anterior Cervical Discectomy and Fusion: A Minimum 2-Year Follow-Up Study. World Neurosurg 2018; 115: e226-e232.

29. Cho HJ, Hur JW, Lee JB, Han JS, Cho TH, Park JY. Cervical stand-alone polyetheretherketone cage versus zero-profile anchored spacer in single-level anterior cervical discectomy and fusion: minimum 2-year assessment of radiographic and clinical outcome. J Korean Neurosurg Soc 2015; 58: 119-124.

30. Shiban E, Nies M, Kogler J, Kogler L, da Cunha PR, Meyer B, Lehmberg J. No correlation between radiological and clinical outcome 1 year following cervical arthrodesis. Acta Neurochir (Wien) 2018; 160 (4): $845-853$.

31. Scholz M, Reyes PM, Schleier P, Sawa A, Baek S, Kandziora F, Marciano F, Crawford N. A new standalone cervical anterior interbody fusion device: Biomechanical comparison with established anterior cervical fixation devices. Spine (Phila Pa 1976) 2009; 34: 156-160.

32. Kast E, Derakhshani S, Bothmann M, Oberle J. Subsidence after anterior cervical inter-body fusion. A randomized prospective clinical trial. Neurosurg Rev 2009; 32: 207-214.

Received January 3, 2019. Accepted March 12, 2019. 